\title{
A GENERALIZATION OF BANACH'S CONTRACTION PRINCIPLE
}

\author{
Lj. B. ĆIRIĆ
}

ABSTRACT. Let $T: M \rightarrow M$ be a mapping of a metric space $(M, d)$ into itself. A mapping $T$ will be called a quasi-contraction iff $d(T x, T y) \leq$ $q \cdot \max \{d(x, y) ; d(x, T x) ; d(y, T y) ; d(x, T y) ; d(y, T x)\}$ for some $q<1$ and all $x, y \in M$. In the present paper the mappings of this kind are investigated. The results presented here show that the condition of quasi-contractivity implies all conclusions of Banach's contraction principle. Multi-valued quasi-contractions are also discussed.

1. Introduction. The well-known Banach's contraction mapping principle states that if $T: M \rightarrow M$ is a contraction on $M$ (i.e. $d(T x, T y) \leq q \cdot d(x, y)$ for some $q<1$ and all $x, y \in M$ ) and $M$ is complete, then

$\left(1^{\circ}\right) T$ has a unique fixed point $\mathbf{u}$ in $M$,

(2०) $\lim _{n} T^{n} x=u$, and

$\left(3^{\circ}\right) d\left(T^{n} x, u\right) \leq q^{n}(1-q)^{-1} d(x, T x)$ for every $x \in M$.

A number of generalizations of this result have appeared [1], [2], [3], [7], [8], [9], [12]. In [2] we considered generalized contractions, defined as follows.

A mapping $T: M \rightarrow M$ is said to be a generalized contraction iff for every $x, y \in M$ there exist nonnegative numbers $q, r, s$ and $t$, which may depend on both $\mathrm{x}$ and $\mathrm{y}$, such that $\sup \{q+r+s+2 t: x, y \in M\}<1$ and

$$
\begin{aligned}
d(T x, T y) \leq & q \cdot d(x, y)+r \cdot d(x, T x) \\
& +s \cdot d(y, T y)+t \cdot[d(x, T y)+d(y, T x)] .
\end{aligned}
$$

S. Nadler [10] has extended Banach's contraction principle to multivalued contractions. Many extensions of Nadler's result have been derived in recent years [4], [6], [11], [13]. In [4] we proved some fixed-point theorems for a class of multi-valued generalized contractions-the maps which include the single-valued generalized contractions.

Received by the editors January 17, 1973.

AMS (MOS) subject classifications (1970). Primary 54E40, 54H25, 47H10; Secondary $54 \mathrm{C} 30$.

Key words and phrases. Quasi-contractions, multi-valued quasi-contractions, fixed-point theorems. 
The purpose of this paper is to extend some results concerning generalized contractions of [2] and [4] to quasi-contractions. In $\$ 2$ fixedpoint theorems for single-valued quasi-contractions are proved and an example is given to show that the results established here are indeed extensions. In $\S 3$ it is shown that for multi-valued quasi-contractions a similar result is valid.

2. Quasi-contractions. Let $T$ be a mapping of a metric space $M$ into itself. For $A \subset M$ let $\delta(A)=\sup \{d(a, b): a, b \in A\}$ and for each $x \in M$, let

$$
\begin{aligned}
& O(x, n)=\left\{x, T x, \cdots, T^{n} x\right\}, \quad n=1,2, \cdots, \\
& O(x, \infty)=\{x, T x, \cdots\} .
\end{aligned}
$$

A space $M$ is said to be $T$-orbitally complete iff every Cauchy sequence which is contained in $O(x, \infty)$ for some $x \in M$ converges in $M$ (cf. [5]).

Definition 1. A mapping $T: M \rightarrow M$ of a metric space $M$ into itself is said to be a quasi-contraction iff there exists a number $q, 0 \leq q<1$, such that

(B) $d(T x, T y) \leq q \cdot \max \{d(x, y) ; d(x, T x) ; d(y, T y) ; d(x, T y) ; d(y, T x)\}$ bolds for every $x, y \in M$.

It is clear that condition (A) implies (B). The following example shows that a quasi-contraction need not be a generalized contraction.

Example. Let

$$
\begin{aligned}
& M_{1}=\{m / n: m=0,1,3,9, \cdots ; n=1,4, \cdots, 3 k+1, \cdots\}, \\
& M_{2}=\{m / n: m=1,3,9,27, \cdots ; n=2,5, \cdots, 3 k+2, \cdots\},
\end{aligned}
$$

and let $M=M_{1} \cup M_{2}$ with the usual metric. Define $T: M \rightarrow M$ by

$$
\begin{aligned}
T x & =3 x / 5, \quad \text { for } x \in M_{1}, \\
& =x / 8, \quad \text { for } x \in M_{2} .
\end{aligned}
$$

The mapping $T$ is a quasi-contraction with $q=3 / 5$. Indeed, if both $\mathbf{x}$ and $\mathbf{y}$ are in $M_{1}$ or in $M_{2}$, then $d(T x, T y) \leq(3 / 5) d(x, y)$. Now let $\mathbf{x}$ be, for example, in $M_{1}$ and $y$ in $M_{2}$. Then

$$
\begin{aligned}
& x>\frac{5}{24} y \text { implies } d(T x, T y)=\frac{3}{5}\left(x-\frac{5}{24} y\right) \leq \frac{3}{5}\left(x-\frac{1}{8} y\right)=\frac{3}{5} d(x, T y) \\
& x<\frac{5}{24} y \text { implies } d(T x, T y)=\frac{3}{5}\left(\frac{5}{24} y-x\right) \leq \frac{3}{5}(y-x)=\frac{3}{5} d(x, y) .
\end{aligned}
$$

Therefore, $T$ on $M$ satisfies the condition 


$$
d(T x, T y) \leq(3 / 5) \max \{d(x, y) ; d(x, T y) ; d(y, T x)\}
$$

and hence (B).

To show that $T$ is not a generalized contraction on $M$, let $x=1$ and $y=1 / 2$. Then we have

$$
\begin{aligned}
q \cdot d(x, y) & +r \cdot d(x, T x)+s \cdot d(y, T y)+t[d(x, T y)+d(y, T x)] \\
= & q \cdot \frac{1}{2}+r \cdot \frac{2}{5}+s \cdot \frac{7}{16}+t \cdot \frac{83}{80} \\
& <(q+r+s+2 t) \cdot \frac{83}{160}<\frac{83}{160}<\frac{43}{80}=d(T x, T y),
\end{aligned}
$$

as $q+r+s+2 t<1$, and we see that condition (A) is not satisfied.

Before stating the fixed-point theorem for quasi-contractions we shall prove two lemmas on these mappings. The first of these lemmas is fundamental.

Lemma 1. Let $T$ be a quasi-contraction on $M$ and let $n$ be any positive integer. Then for each $x \in M$ and all positive integers $i$ and $j, i, j \epsilon$ $\{1,2, \cdots, n\}$ implies $d\left(T^{i} x, T^{j} x\right) \leq q \cdot \delta[O(x, n)]$.

Proof. Let $x \in M$ be arbitrary, let $\mathbf{n}$ be any positive integer and let $\mathbf{i}$ and $\mathbf{j}$ satisfy the condition of Lemma 1 . Then $T^{i-1} x, T^{i} x, T^{j-1} x, T^{j} x \in$ $O(x, n)$ (where it is understood that $T^{0} x=x$ ) and since $T$ is a quasiocontraction, we have

$$
\begin{array}{rlrl}
d\left(T^{i} x, T^{j} x\right) & =d\left(T T^{i-1} x, T T^{j-1} x\right) \\
& \leq q \cdot \max \left\{d\left(T^{i-1} x, T^{j-1} x\right) ; d\left(T^{i-1} x, T^{i} x\right) ; d\left(T^{j-1} x, T^{j} x\right) ;\right. \\
& \leq q \cdot \delta[O(x, n)], & \left.d\left(T^{i-1} x, T^{j} x\right) ; d\left(T^{i} x, T^{j-1} x\right)\right\}
\end{array}
$$

which proves the lemma.

Remark. From this lemma it follows that if $T$ is a quasi-contraction and $x \in M$, then for every positive integer $\mathbf{n}$ there exists a positive integer $k \leq n$, such that $d\left(x, T^{k} x\right)=\delta[O(x, n)]$.

Lemma 2. If $T$ is a quasi-contraction on $M$, then

$$
\delta[O(x, \infty)] \leq(1 /(1-q)) d(x, T x)
$$

holds for all $x \in M$.

Proof. Let $x \in M$ be arbitrary. Since $\delta[O(x, 1)] \leq \delta[O(x, 2)] \leq \cdots$, we 
see that $\delta[O(x, \infty)]=\sup \{\delta[O(x, n)]: n \in N\}$. The lemma will follow if we show that $\delta[O(x, n)] \leq(1 /(1-q)) d(x, T x)$ for all $n \in N$.

Let $\mathbf{n}$ be any positive integer. From the remark to the previous lemma, there exists $T^{k} x \in O(x, n)(1 \leq k \leq n)$ such that $d\left(x, T^{k} x\right)=\delta[O(x, n)]$. Applying a triangle inequality and Lemma 1 , we get

$$
\begin{aligned}
d\left(x, T^{k} x\right) & \leq d(x, T x)+d\left(T x, T^{k} x\right) \leq d(x, T x)+q \cdot \delta[O(x, n)] \\
& =d(x, T x)+q \cdot d\left(x, T^{k} x\right) .
\end{aligned}
$$

Therefore, $\delta[O(x, n)]=d\left(x, T^{k} x\right) \leq(1 /(1-q)) d(x, T x)$. Since $\mathbf{n}$ was arbitrary, the proof is completed.

Now we can state our main result.

Theorem 1. Let $T$ be a quasi-contraction on a metric space $M$ and let $M$ be T-orbitally complete. Then

(a) $T$ has a unique fixed point $\mathbf{u}$ in $M$,

(b) $\lim _{n} T^{n} x=u$, and

(c) $d\left(T^{n} x, u\right) \leq\left(q^{n} /(1-q)\right) d(x, T x)$ for every $x \in M$.

Proof. Let $\mathbf{x}$ be an arbitrary point of $M$. We shall show that the sequence of iterates $\left\{T^{n} x\right\}$ is a Cauchy sequence.

Let $\mathbf{n}$ and $\mathbf{m}(n<m)$ be any positive integers. Since $T$ is a quasi-contraction, it follows from Lemma 1 that

$$
d\left(T^{n} x, T^{m} x\right)=d\left(T T^{n-1} x, T^{m-n+1} T^{n-1} x\right) \leq q \cdot \delta\left[O\left(T^{n-1} x, m-n+1\right)\right] .
$$

According to the remark to Lemma 1 , there exists an integer $k_{1}, 1 \leq k_{1} \leq$ $m-n+1$, such that

$$
\delta\left[O\left(T^{n-1} x, m-n+1\right)\right]=d\left(T^{n-1} x, T^{k} T^{n-1} x\right) .
$$

Again, by Lemma 1, we have

$$
\begin{aligned}
d\left(T^{n-1} x, T^{k}{ }^{1} T^{n-1} x\right) & =d\left(T T^{n-2} x, T^{k+1} T^{n-2} x\right) \\
& \leq q \cdot \delta\left[O\left(T^{n-2} x, k_{1}+1\right)\right] \\
& \leq q \cdot \delta\left[O\left(T^{n-2} x, m-n+2\right)\right] .
\end{aligned}
$$

Therefore, we have the following system of inequalities.

$$
d\left(T^{n} x, T^{m} x\right) \leq q \cdot \delta\left[O\left(T^{n-1} x, m-n+1\right)\right] \leq q^{2} \cdot \delta\left[O\left(T^{n-2} x, m-n+2\right)\right] .
$$

Proceeding in this manner, we obtain 


$$
d\left(T^{n} x, T^{m} x\right) \leq q \cdot \delta\left[O\left(T^{n-1} x, m-n+1\right)\right] \leq \cdots \leq q^{n} \cdot \delta[O(x, m)] .
$$

Then it follows from Lemma 2 that

$$
d\left(T^{n} x, T^{m} x\right) \leq\left(q^{n} /(1-q)\right) d(x, T x) .
$$

Since $\lim _{n} q^{n}=0,\left\{T^{n} x\right\}$ is a Cauchy sequence.

Again, $M$ being $T$-orbitally complete, $\left\{T^{n} x\right\}$ has a limit $\mathbf{u}$ in $M$. To prove that $T u=u$, let us consider the following inequalities.

$$
\begin{aligned}
d(u, T u) \leq d\left(u, T^{n+1} x\right)+d\left(T T^{n} x, T u\right) & \\
\leq d\left(u, T^{n+1} x\right)+q \cdot \max \left\{d\left(T^{n} x, u\right),\right. & d\left(T^{n} x, T^{n+1} x\right) ; \\
\left.d(u, T u) ; d\left(T^{n} x, T u\right) ; d\left(T^{n+1} x, u\right)\right\} & \\
\leq d\left(u, T^{n+1} x\right)+q \cdot\left[d\left(T^{n} x, T^{n+1} x\right)\right. & +d\left(T^{n} x, u\right) \\
& \left.+d(u, T u)+d\left(T^{n+1} x, u\right)\right] .
\end{aligned}
$$

Hence

$d(u, T u) \leq \frac{1}{1-q}\left[(1+q) d\left(u, T^{n+1} x\right)+q \cdot d\left(u, T^{n} x\right)+q \cdot d\left(T^{n} x, T^{n+1} x\right)\right]$.

Since $\lim _{n} T^{n} x=u$, this shows that $d(u, T u)=0$, i.e. $\mathbf{u}$ is a fixed point under $T$. The uniqueness follows from the quasi-contractivity of $T$. So we have proved (a) and (b), as $x$ was arbitrary. Letting $m$ tend to infinity in (1), we obtain the inequality (c).

This completes the proof of the theorem.

The next result readily follows from the above theorem.

Theorem 2. Let $T$ be a mapping of a metric space $M$ into itself and let $M$ be T-orbitally complete. If there exists a positive integer $k$ such that the iteration $T^{k}$ is a quasi-contraction, then

( ${ }^{\prime}$ ) $T$ has a unique fixed point $\mathbf{u}$ in $M$,

(b') $\lim _{n} T^{n} x=u$, and

(c') $d\left(T^{n} x, u\right) \leq q^{m} a(x) /(1-q)$ for every $x \in M$, where $a(x)=\max \left\{d\left(T^{i} x, T^{i+k} x\right): i=0,1, \cdots, k-1\right\}$ and $m=E(n / k)$ is the greatest integer not exceeding $n / k$.

Proof. Since $T^{k}$ has a unique fixed point $\mathbf{u}$ and $T^{k}(T u)=T\left(T^{k} u\right)=T u$, it follows that $T u=u$. Its uniqueness is obvious. To show ( $\left.c^{\prime}\right)$, let $\mathbf{n}$ be any integer. Then $\mathbf{n}=m \cdot k+j, 0 \leq j<k, m \geq 0$, and for every $x \in M, T^{n} x=$ $\left(T^{k}\right)^{m} T^{j} x$. Since $T^{k}$ is a quasi-contraction, it follows from part (c) of Theorem 1 that 


$$
\begin{aligned}
d\left(T^{n} x, u\right) & \leq \frac{q^{m}}{1-q} d\left(T^{j} x, T^{k} T^{j} x\right) \\
& \leq \frac{q^{m}}{1-q} \max \left\{d\left(T^{i} x, T^{k} T^{i} x\right): i=0,1, \cdots, k-1\right\},
\end{aligned}
$$

which proves $\left(c^{\prime}\right)$, and hence $\left(b^{\prime}\right)$. This completes the proof of the theorem.

Note that Theorem 2.5 (Theorem 2.6) of [2] is a special case of Theorem 1 (Theorem 2). The example following Definition 1 shows that Theorem 1 is more general than Theorem 2.5 of [2]. In that example $M$ is T-orbitally complete and $o$ is a fixed point under $T$.

3. Multi-valued quasi-contractions. We shall now recall some terminologies. Let $(M, d)$ be a metric space and let $A, B$ be any subsets of $M$. We denote $D(A, B)=\inf \{d(a, b): a \in A, b \in B\}, \rho(A, B)=\sup \{d(a, b): a \in A, b \in B\}$, $B N(M)=\{A: \varnothing \neq A \subset M$ and $\delta(A)<+\infty\}$. Let $F: M \rightarrow M$ be a point to set correspondence and let $x_{0} \in M$. An orbit of $F$ at $x_{0}$ is a sequence $\left\{x_{n}: x_{n} \in F x_{n-1}, n=1,2, \cdots\right\}$. A space $M$ is said to be F-orbitally complete iff every Cauchy sequence which is a subsequence of an orbit of $F$ at $\mathbf{x}$ for some $x \in M$, converges in $M$. Among the results established in [4] was the following: if $F: M \rightarrow B N(M)$ satisfies

(C) $\rho(F x, F y) \leq q \cdot \max \{d(x, y) ; \rho(x, F x) ; \rho(y, F y) ; 1 / 2[D(x, F y)+D(y, F x)]\}$

for some $q<1$ and if $M$ is $F$-orbitally complete, then $F$ has a unique fixed point $\mathbf{u}$ with $F u=\{u\}$ and for each $x_{0} \in M$ there exists an orbit $\left\{x_{n}\right\}$ of $F$ at $x_{0}$ such that $\lim _{n} x_{n}=u$. The following is an extension of the above statement.

Theorem 3. Let $F: M \rightarrow B N(M)$ be a multi-valued mapping on a metric space $M$ and let $M$ be F-orbitally complete. If $F$ satisfies

(D) $\rho(F x, F y) \leq q \cdot \max \{d(x, y) ; \rho(x, F x) ; \rho(y, F y) ; D(x, F y) ; D(y, F x)\}$ for some $q<1$ and all $x, y \in M$, then

(i) $F$ has a unique fixed point $\mathbf{u}$ in $M$ and $F u=\{u\}$,

(ii) for each $x_{0} \in M$ there exists an orbit $\left\{x_{n}\right\}$ of $F$ at $x_{0}$ such that $\lim _{n} x_{n}=u$, and

(iii) $d\left(x_{n}, u\right) \leq\left(\left(q^{1-a}\right)^{n} /\left(1-q^{1-a}\right)\right) d\left(x_{0}, x_{1}\right)$, where $a<1$ is any fixed positive number.

Proof. Let $a \in(0,1)$ be any number. Define a single-valued mapping $T: M \rightarrow M$ as follows: for each $x \in M$ let $T x$ be a point of $F x$, which satisfies 
$d(x, T x) \geq q^{a} \cdot \rho(x, F x)$. A mapping $T$ is then a quasi-contraction with $q_{1}=$ $q^{1-a}$. Indeed, for every $x, y \in M$ we have

$$
d(T x, T y) \leq \rho(F x, F y)
$$

$$
\begin{gathered}
\leq q \cdot q^{-a} \max \left\{q^{a} d(x, y) ; q^{a} \rho(x, F x) ; q^{a} \rho(y, F y) ;\right. \\
\left.q^{a} D(x, F y) ; q^{a} D(y, F x)\right\} \\
\leq q^{1-a} \max \{d(x, y) ; d(x, T x) ; d(y, T y) ; d(x, T y) ; d(y, T x)\},
\end{gathered}
$$

which means that $T$ is a quasi-contraction. Clearly $u=T u$ implies $u \in F u$. Since $F$ satisfies (D), $u \in F u$ implies $\rho(F u, F u) \leq q \cdot \rho(u, F u)$. This may happen only if $F u=\{u\}$. Therefore, $u \in M$ is a fixed point of $T$ iff $\mathbf{u}$ is a fixed point of $F$. Since for each $x \in M$ the sequence $\left\{T^{n} x\right\}$ is an orbit of $F$ at $x$, the statements of Theorem 3 follow from Theorem 1 .

\section{REFERENCES}

1. V. W. Bryant, A remark on a fixed point theorem for iterated mappings, Amer. Math. Monthly, 75 (1968), 399-400. MR 37 \#2210.

2. Lj. Cirić, Generalized contractions and fixed-point theorems, Publ. Inst. Math. 12 (26) (1971), 19-26. MR 46 \#8203.

3. - Fixed point theorems for mappings with a generalized contractive iterate at a point, Publ. Inst. Math. 13 (27) (1972), 11-16.

4. - Fixed points for generalized multi-valued contractions, Mat. Vesnik 9 (24) (1972), 265-272.

5. - On contraction type mappings, Math. Balkanica 1 (1971), 52-57.

6. L. Dube and S. Singh, On multi-valued contraction mappings, Bull. Math. Soc. Math. RSR 14 (1970), 307-310 (1971).

7. M.: Edelstein, An extension of Banach's contraction principle, Proc. Amer. Math. Soc. 12 (1961), 7-10. MR 22 \#11375.

8. L. F. Guseman, Jr., Fixed point theorems for mappings with a contractive iterate at a point, Proc. Amer. Math. Soc. 26 (1970), 615-618. MR 42 \#919.

9. R. Kannan, Some results on fixed points. II, Amer. Math. Monthly 76 (1969), 405-408. MR $41 \# 2487$.

10. S. B. Nadler, Jr., Multi-valued contraction mappings, Pacific J. Math. 30 (1969), 475-488. MR $40 \# 8035$.

11. S. Reich, Fixed points of contractive functions, Bol. Un. Mat. Ital. (4) 5 (1972), 26-42.

12. V. M. Sehgal, A fixed point theorem for mappings with a contractive iterate, Proc. Amer. Math. Soc. 23 (1969), 631-634. MR 40 \#3531.

13. R. Smithson, Fixed points for contractive multifunctions, Proc. Amer. Math. Soc. 27 (1971), 192-194. MR 42 \#2466. 